\title{
Performance Analysis of Coal Cleaning Operations: Relationship Between Probable Error in Separation and Organic Efficiency Performance Analysis of Coal Cleaning Operations: Relationship Between Probable Error in Separation and Organic Efficiency
}

Subha Ranjan Paul ( $\square$ spaul394@gmail.com )

Aditya Engineering College

Sumantra Bhattacharya

IIT (ISM): Indian Institute of Technology

Research

Keywords: Organic Efficiency, Ecart Probable, Dichotomy, Plant Performance, Jigs

Posted Date: April 21st, 2021

DOI: https://doi.org/10.21203/rs.3.rs-442912/v1

License: (c) (1) This work is licensed under a Creative Commons Attribution 4.0 International License.

Read Full License 


\title{
Performance Analysis of Coal Cleaning Operations: Relationship between Probable Error in Separation and Organic Efficiency
}

\author{
*Subha Ranjan Paul and **Sumantra Bhattacharya \\ *Department of Mining Engineering, Aditya Engineering College, Surampalem, Andhra Pradesh, India-533437 \\ **Department of Fuel \& Mineral Engineering, Indian Institute of Technology (Indian School of Mines), Dhanbad, \\ Jharkhand, India - 826004
}

\section{Corresponding Author Mail id :- subhapau12405@gmail.com}

\begin{abstract}
Separation efficiency of coal cleaning equipment is typically assessed by Probable Error in Separation $\left(\mathrm{E}_{\mathrm{p}}\right)$ and Organic Efficiency $\left(\mathrm{E}_{\mathrm{org}}\right)$. The first one is determined on the basis of precise cut point density of separation and implies that for ideal separation the error is zero. The second one is calculated on the basis of yield of clean coal/ middling at the target ash and implies that for ideal separation the efficiency is $100 \%$. Plant operators worldwide being accountable for the tonnage of the clean coal and middling produced regularly monitor $\mathrm{E}_{\text {org }}$ with some application in plant design in India. $E_{p}$ is universally used as an equipment selection criterion from among the vendors, in commercial contracts and sometimes for performance analysis of coal cleaning equipment carried out at the plants. Since both are performance measures there should possibly be a relationship between the two for specific cleaning equipment or for a particular type of density separators. Such relationships are however rarely observed.

Moreover there are many instances where high to very high $\mathrm{E}_{\mathrm{org}}$ does not translate into low to very low $\mathrm{E}_{\mathrm{p}}$.

Therefore, is there a dichotomy between the two performance measures?
\end{abstract}

Key words : Organic Efficiency, Ecart Probable, Dichotomy, Plant Performance, Jigs

\section{Introduction}

Separation efficiency of coal cleaning equipment is usually assessed by the following measures.

O Tromp curves, also known as partition curves and distribution curves, which compare the ideal and actual separation curves and the difference, if any, arising between the two curves known as Error Area

- $E_{\mathrm{p}}$ (Ecart Probable Moyen - EPM) also known as Probable Error in Separation: mathematically defined as:

$$
E_{p}=\left(\longleftarrow d^{25}-{ }_{2} d^{75}\right) ; E_{p}=\left(\longleftarrow d 75-{ }_{2} d 25\right)
$$

Where, $d_{25}$ and $d_{75}$ indicate $25 \%$ and $75 \%$ probability respectively, also known as partition numbers, for the particles to report to a given cleaning product. The dimensionless value of $\mathrm{E}_{\mathrm{p}}$ attempts to capture the shift of the actual partition curve from the ideal curve (Fig. 1) by quantifying the slope of the partition curve that indicates the sharpness of separation achieved. For an ideal separation, $\mathrm{E}_{\mathrm{p}}=0$

- Percentage misplacement of clean coal to reject

- Percentage misplacement of reject to clean coal

O Percentage actual (total) misplacement defined as the total amount of material misplaced in both the

Figure 1 Typical partition curve 
sides of cut density, calculated from partition curve or obtained from the float - sink data used to plot the partition curve or from the single point float - sink test at the cut density. The Ep is made independent of cut densities by using Imperfection (I).

- $\quad I=E p / d_{50}$, for Dense Media (DM) processes

O $\mathrm{I}=\mathrm{Ep} /\left[\mathrm{d}_{50}-1\right]$, for water based separations, such as in Jigs, Spirals etc For an ideal separation, since $\mathrm{E}_{\mathrm{p}}=0$, also $\mathrm{I}=0$.

O Organic Efficiency, $E_{\text {org }}$, defined as the ratio of the actual yield at the plant to the washability yield at the same ash, expressed in percentage. For an ideal separation, $\mathrm{E}_{\text {org }}=100 \%$ [Bhattacharya et al, 2016]

In a Dense Media (DM) separator, there is a difference, known as "offset" between the medium relative density (RD) and the cut density, $\mathrm{d}_{50}$. Possibly RD of the medium among others contributes most to the "offset". The "offset" is generally positive and is rather less in DM vessels. Typically $\mathrm{d}_{50}$ of a DMC is higher than the feed medium RD by up to 0.15 RD units (Fig. 2). RD "offset" also takes place even in Jigs and Spirals [Sanders, 2007]. "Offset" is usually positive. In case of Jigs, the "offset" could be on both sides. While setting the cut point density for any coal cleaning equipment, if "offset" is not given due consideration, error in separation could be large.

Plant operators worldwide usually prefer for day to day monitoring organic efficiency and the three misplacement parameters. Being accountable for the tonnage of the clean coal and middling produced preference towards easily determined organic efficiency is possibly because that provides them incentives to maximize the yields of clean coal and middling and minimize the yield of reject. Ep is universally used as an equipment selection criterion from among the vendors, in commercial contracts in plant commissioning and handover and is very popular among technology providers and plant operators as a key performance guarantee parameter. "Ep" and "I" are less frequently used in plants as a tool for performance evaluation of coal cleaning equipment [Laurilla, 1998]. Since most of these are density based separators, researchers, process analysts worldwide put more emphasis on Ep possibly because that involves precise determination of the cut point density of separation.

Figure 2 Relationship between DMC feed media density and cut density after Stamicarbon [Sanders, 2007]

\section{Effect of Ep and $E_{\text {org }}$ on Plant Performance}

Hundred percent organic efficiency and zero probable error are rarely achieved, particularly the latter one. Table 1 shows variation of clean coal yield and ash with probable error in separation. To further emphasize the significance of Ep, consider a 500tph plant with a clean coal target ash of 10\%. The difference in product tonnage between an Ep of 0.00 and 0.03 is $(75.0-72.3) \times(500 / 100)=13.5$ tph. If the plant operates for 330days in a year and a moderate 18hours per day, then the clean coal production loss per year is $13.5 \times 18 \times 330=80190 t$. If we assume Rs6000/ $t$ is, the basic price of this metallurgical (met) coal then the revenue loss is Rs48.114crores per year.

Table 1 Variation of yield and ash with probable error in separation [Sanders, 2007] 
Similar, yet more direct calculation of revenue loss can be done on the basis of just $1 \%$ drop from guaranteed organic efficiency value. Consider a 300tph plant with the following cleaning parameters as obtained from washability study (Table 2). If the plant operates for 330days in a year and same 18hours per day, then the clean coal production loss per year is $2.4 \times 18 \times 330=14256 t$. If we assume Rs $2000 / t$ is, the basic price of this thermal coal then the revenue loss is Rs 2.85 crores per year.

\section{Table 2 Thermal coal cleaning parameters}

$100-96=4 \%$ loss in organic efficiency implies $4 \%$ of recoverable clean coal gets misplaced in reject leading to loss of combustibles. 5\% (68-63.2=4.8) ash drop in plant generated reject appears to be equivalent to a GCV loss of $150200 \mathrm{kcal} / \mathrm{kg}$ for Indian thermal coal. In spite of certain limitations, organic efficiency therefore provides lot of information useful for the day to day operation of the plants. Limitations include the following.

It is silent on reject misplaced in clean coal

Average clean coal ash of $33 \%$ can be the outcome of numerous permutations and combinations of individual particle ash, e.g. $(66+0) / 2=33 ;(50+16) / 2=33 ;(40+26) / 2=33$ and so on, therefore it is also silent on low ash clean coal misplaced in reject.

\section{Contradicting Performance Results for Ep and $E_{o r g}$}

Partition curve and calculated from that probable error in separation are however quite scientific since the measures involve precise determination of cut density, the very basis of separation and the error in separation around that. Since probable error in separation and organic efficiency, both are performance measures there should possibly be a relationship between the two for specific cleaning equipment or for a particular type of density separators. Such relationships are however rarely observed. There are many instances where high to very high $\mathrm{E}_{\text {org }}$ does not translate into low to very low $\mathrm{E}_{\mathrm{p}}$. Table 3 shows typical Ep values for some of the density separators used in coal cleaning.

Table 3 Ep for some of the density separation processes [Gupta and Yan, 2016]

Table 4 shows efficiency parameters of cleaning units treating Jharia coal. DM Bath has Ep value very close to the best one (Table 3), yet organic efficiency of $98 \%$ could have been better. Baum Jig shows high organic efficiency of close to $97 \%$, just about $1 \%$ less than that achieved for DM Bath. Yet, Ep is seven times more than that obtained for DM Bath. DMC records reasonable Ep (Table 5), though inferior $E_{\text {org. }}$. Table 4 shows feed size, cut density and NGM [near gravity material] are well within the prescribed range for all the three equipment [Bhattacharya et al, 2016]. $E_{p}$ values recommended by Australian Coal Preparation Society (Table 5) are justified by Fig. 3 showing consistent rise in $E_{p}$ values with the increase in cut density irrespective of particle size.

Table 4 Cleaning equipment performance for Jharia coal 
Figure 3 Relationship between probable error, cut density and particle size for DMC [Sanders, 2007]

Table 6 shows size by size performance analysis of a Baum Jig treating $<75 \mathrm{~mm}$ sized raw coal. Performance consistently deteriorates with increase in cut density and decrease in particle size for the constituent size fractions of the composite feed to the extent that for the finest two fractions, probable error in separation and imperfection could not be determined. Comparing the determined values it is observed that the coarsest fraction of $>25 \mathrm{~mm}$ has half the Ep value of that obtained for the size $1.6 \times 1.0 \mathrm{~mm}$, all the values being within the prescribed range (Table 3 ). In contrast for two similar South African coals organic efficiency of Jigs consistently increases with the rise in cut density for one coal achieving the value of $100 \%$ at a cut density of approximately 1.97 (Fig. 4).

Table 5 Recommended Ep values for DMC operation [Sanders, 2007]

Table 6 Performance of a Baum Jig treating composite <75mm feed [Sanders, 2007]

Figure 4 Changes in organic efficiency of Jigs with cut density [Horsfall,1987]

\section{Selected Indian Case Studies on Jigs}

In a rare case of conformity, on the basis of size by size performance analysis, Fig. 5 and Fig. 6 both show considerable improvement in imperfection and organic efficiency, respectively with the rise in cut-density for Chasnala Batac Jig. Imperfection in fact has a reasonably good empirical relationship with cut-density. In case of some of the size fractions, organic efficiency achieves highest possible values or very close to that, though corresponding imperfection values fall short.

Figure 5 Dependency of imperfection on cut-density for Chasnala Batac Jig

Figure 6 Dependency of organic efficiency on cut-density for Chasnala Batac Jig

Fig. 7 and 8 show that for Sharshetali Batac Jig Ep and I rather steadily increase with the rise in cut-density indicating poor separation. At the same time, though in an erratic manner, organic efficiency also increases with cutdensity (Fig. 9) signifying good separation. Most of the values are in the range of 95-99.5\%. As a result no relationship emerges between probable error in separation and organic efficiency (Fig. 10). Fig. 11 shows a reasonable relationship between particle size and organic efficiency for Sharshetali Batac Jig where the latter improves with the increase in the former. In a composite feed of $100 x 0 \mathrm{~mm}$, particles with mean size greater than $32 \mathrm{~mm}$ essentially record organic efficiency in the range of $92-100 \%$.

Figure 7 Plot between probable error in separation and cut-density for Sharshetali Batac Jig: Coal 1

Figure 8 Plot between imperfection and cut-density for Sharshetali Batac Jig: Coal 1 
Composite performance analysis results for two different Batac Jigs cleaning three different coal streams show (Table 7) organic efficiency increases with the rise in cut density. In case of Sharshetali Batac Jig, cut density increase by 0.1 leads to $2 \%$ improvement in organic efficiency, $97 \%$ value being quite close to the ideal one.

\section{Examples from Dense Media Bath Operations}

Table 8 shows performance results for typical DM Bath practices in Canadian and South African coal cleaning plants. In most of the cases small values of probable error in separation and imperfection are accompanied by large values of organic efficiencies. Only exception is Canadian plant A, where very high organic efficiency is not matched by a moderate value of probable error in separation.

Figure 9 Plot between organic efficiency and cut-density for Sharshetali Batac Jig: Coal 1

Figure 10 Plot between organic efficiency and Ep for Sharshetali Batac Jig: Coal 1

Figure 11 Size by size organic efficiencies for Sharshetali Batac Jig: Coal 2 Table 7 Composite performance analysis results for two different Batac Jigs

Table 9 shows performance parameters for the Bhojudih DM Leebar bath. The composite feed has a wide size distribution. With the decrease in size of the constituent particles, cut density increases along with the increase in clean coal yield and ash. Last row of the table shows composite values on weighted average basis. Very high yield of clean coal, close to $79 \%$ is obtained at a reasonable ash content of $17.8 \%$. Reject ash, just short of $60 \%$, is also within acceptable limit. Composite cut density of 1.69 is not unusual for pre-cleaning baths. With the decrease in particle size organic efficiency drops which is not unexpected from dense media separation, yet composite value achieved is quite high and typical for a DM bath, 98\%. In addition, organic efficiency shows quite high coefficient of correlation with cut density and particle size (Fig. 12-13). Ep and I however keep on increasing substantially with the decrease in particle size and corresponding rise in cut density, achieving composite values inferior to the values typical even for pre-cleaning baths. Between the coarsest and finest sizes for 1.7\% drop in organic efficiency Ep and I deteriorate by 5.7times and 40times, respectively.

It is evident therefore, that for both DM Baths and Jigs probable error in separation and Organic Efficiency do not produce matching trends and values in performance analysis. This is particularly true for Jigs. The question that obviously arises is which performance measure to use for the evaluation of Jigs! It is an important question because Jigs are already the most commonly used processing units in thermal coal cleaning in India. New BCCL plants of Dahibari and Patherdih I also have pre-cleaning Jigs as the primary treatment units because of high ash content of the ROM met coal. As indicated by Table 10 Jigs are expected to be the cleaning units in all the plants listed because of high to very high cut density requirement. 
Table 8 Typical DM Bath practices in Canadian and South African plants [Horsfall, 1987]

Table 9 Size by size cleaning parameters for the Bhojudih Leebar bath [Behera, Bhattacharya, 2018]

Feed size distribution appears to have considerable effect on the performance of the Baum or Batac Jigs. Possibly because of size effect, no meaningful separation could be achieved in the Jigs worldwide for particles smaller than $6 \mathrm{~mm}$ or $4 \mathrm{~mm}$ or $2 \mathrm{~mm}$ (Table 6; Fig. 11). As it appears in Jigs treating composite feeds, for finer particles hydrodynamic forces become significantly more dominant than gravity forces [SACPS, 2011]. Possibly as a result particles get kind of fluidized and report to wherever the fluids carry them. Rong and Lyman [1992] found jigging time to be a very significant parameter affecting the bed stratification. In general bed stratification improved quickly up to a jigging time of 180 s, and thereafter slowly. When the jigging time exceeded 300 s, the bed was in a state of dynamic equilibrium for material larger than $8 \mathrm{~mm}$; however, the particles $<8 \mathrm{~mm}$ needed more time to achieve such a state. They had used a pilot scale Baum Jig. Commercial Jigs however operate on the basis of an average jigging time.

Figure 12 Dependency of organic efficiency on cut-density for the Bhojudih Leebar bath [Behera, Bhattacharya, 2018]

Figure 13 Dependency of organic efficiency on particle size for the Bhojudih Leebar bath [Behera, Bhattacharya, 2018]

Table 10 Jigs in proposed thermal coal cleaning plants of CIL [SECL; MCL; CCL, 2018]

\section{Conclusion}

Unlike dense media systems where cut density is imposed, Jigs, Spirals, Barrel Washers, Wilfley Tables are water based separation processes where cut density is self generated by the process in specific equipment. That possibly explains why cut density based performance measures such as Ecart probable error and Imperfection may not always reflect the true performance of the Jigs. Instead organic efficiency which is based on relative movement of particles between the products appears to reflect the true performance of the Jigs.

Therefore, is there a dichotomy between the two performance measures? Certainly that is not, because basis of determination is different. Organic Efficiency however appears to reflect more authentically the Jig performance. What should be the minimum performance guarantee value for Jigs? Possibly that should be $96 \%$ and even $97 \%$.

\section{References}

Behera, Dibya Ranjan, Bhattacharya, S., (2018), Size by Size Performance Analysis of a Leebar Dense Media Bath, International Journal of Coal Preparation and Utilization, https//doi.org/10.1080/19392699.2018.1540983 
Bhattacharya , S., Maheshwari, Abhishek, Panda, Manishankar , (2016), Coal cleaning operations - The Question of Near Gravity Material, Transactions Indian Institute of Metals, 69 (1), pp 157-172

CCL: Central Coalfields Ltd, RFQ \& RFP for 7.0Mtpy Konar Washery

Gupta A, Yan D, 2016, Mineral Processing Design and Operations 2nd Edition, Elsevier, pp 545 (2016)(Table 15.13) Horsfall, D. W., (1987), The Lows and Highs of Dense Medium Separation, The Australian Institute of Mining and Metallurgy Southern Queensland Branch, Dense Medium Operators' Conference

Laurila, M. J., (1998),Best Practices in International Coal Preparation: A Benchmarking Study, X International Coal Preparation Congress Vol. I, Brisbane, pp 17-24

MCL: Mahanadi Central Coalfields Ltd, RFQ \& RFP for 10.0Mtpy Hingula Washery

Rong, R.X., Lyman, G.J, (1992), The Effect of Jig Operating Parameters on Bed Stratification in a Pilot Scale Baum Jig, Fuel, 71, pp. 115-123

Sanders Joe, (Ed.), (2007), Chapter 9 Gravity Separation - Jigs in The Principles of Coal Preparation, $4^{\text {th }}$ edition, Australian Coal Preparation Society, New Castle

SACPS; South African Coal Processing Society; (2011), Coal Preparation in South Africa, 154-228.

SECL: South Eastern Coalfield Ltd, (2018), NIT No: GM (C)/ SECL/ BSP/ eTender/ KSM/113, 
Figures

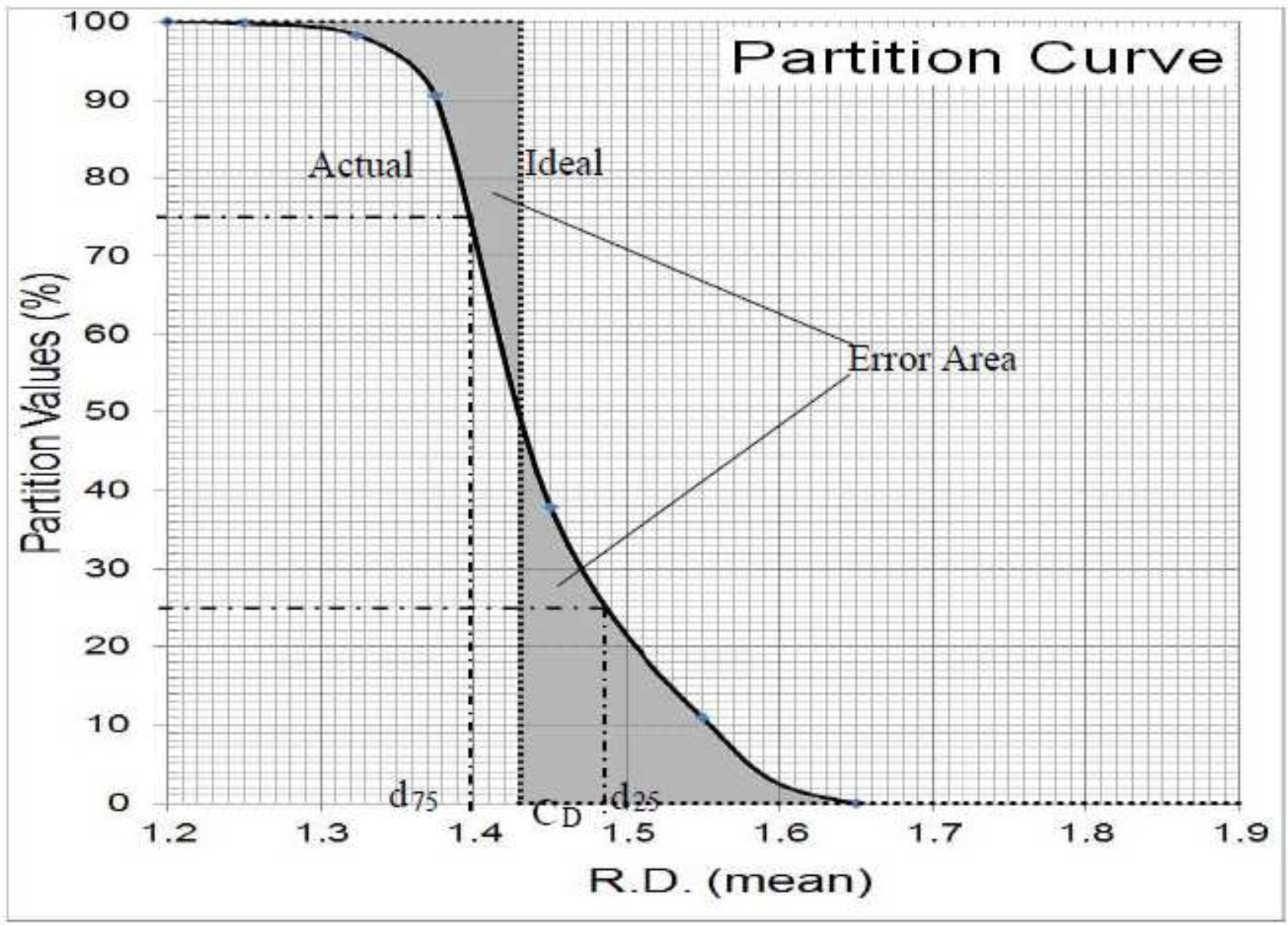

Figure 1

Typical partition curve 


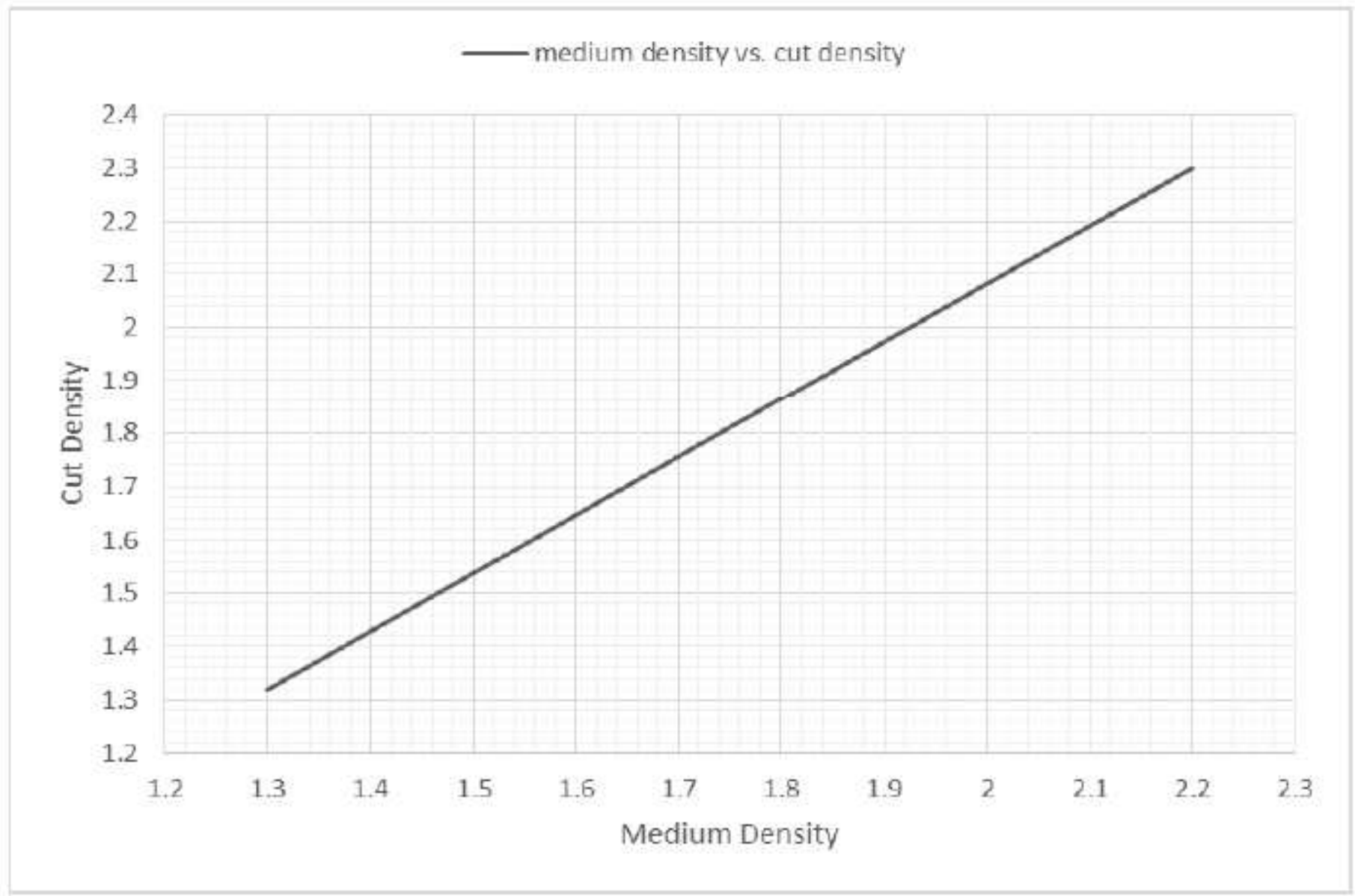

Figure 2

Relationship between DMC feed media density and cut density after Stamicarbon [Sanders, 2007]

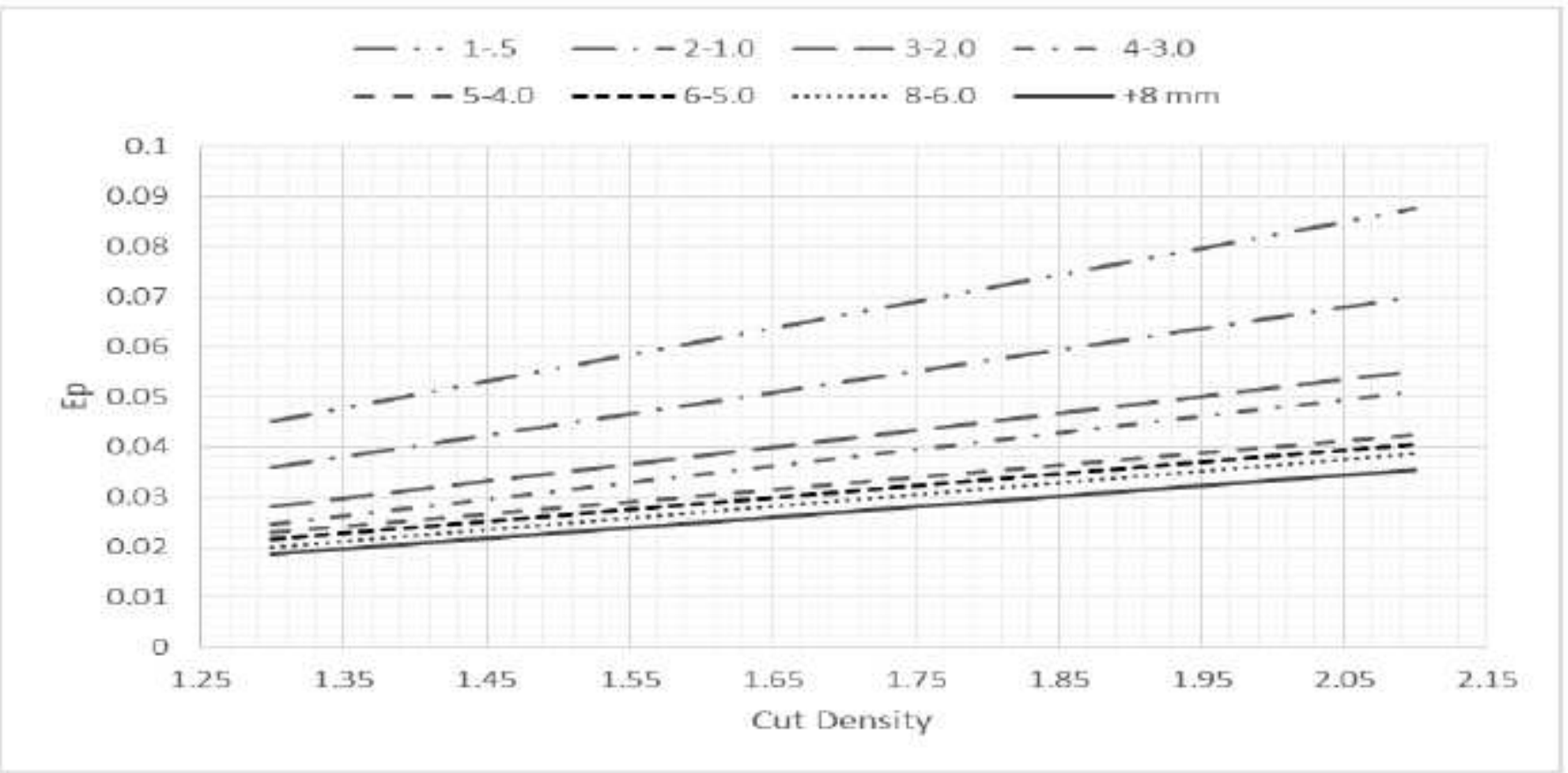

\section{Figure 3}


Relationship between probable error, cut density and particle size for DMC [Sanders, 2007]

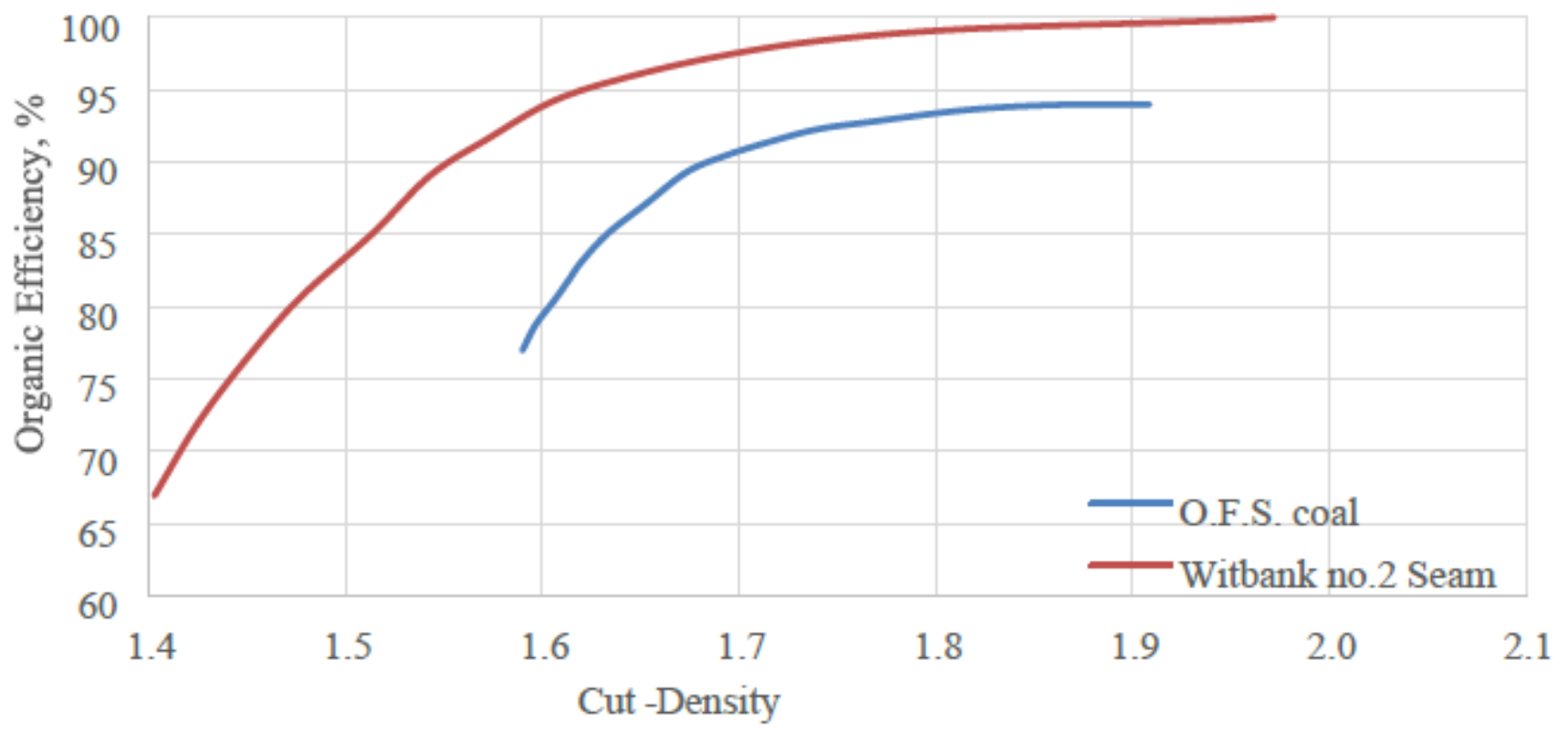

Figure 4

Changes in organic efficiency of Jigs with cut density [Horsfall,1987]

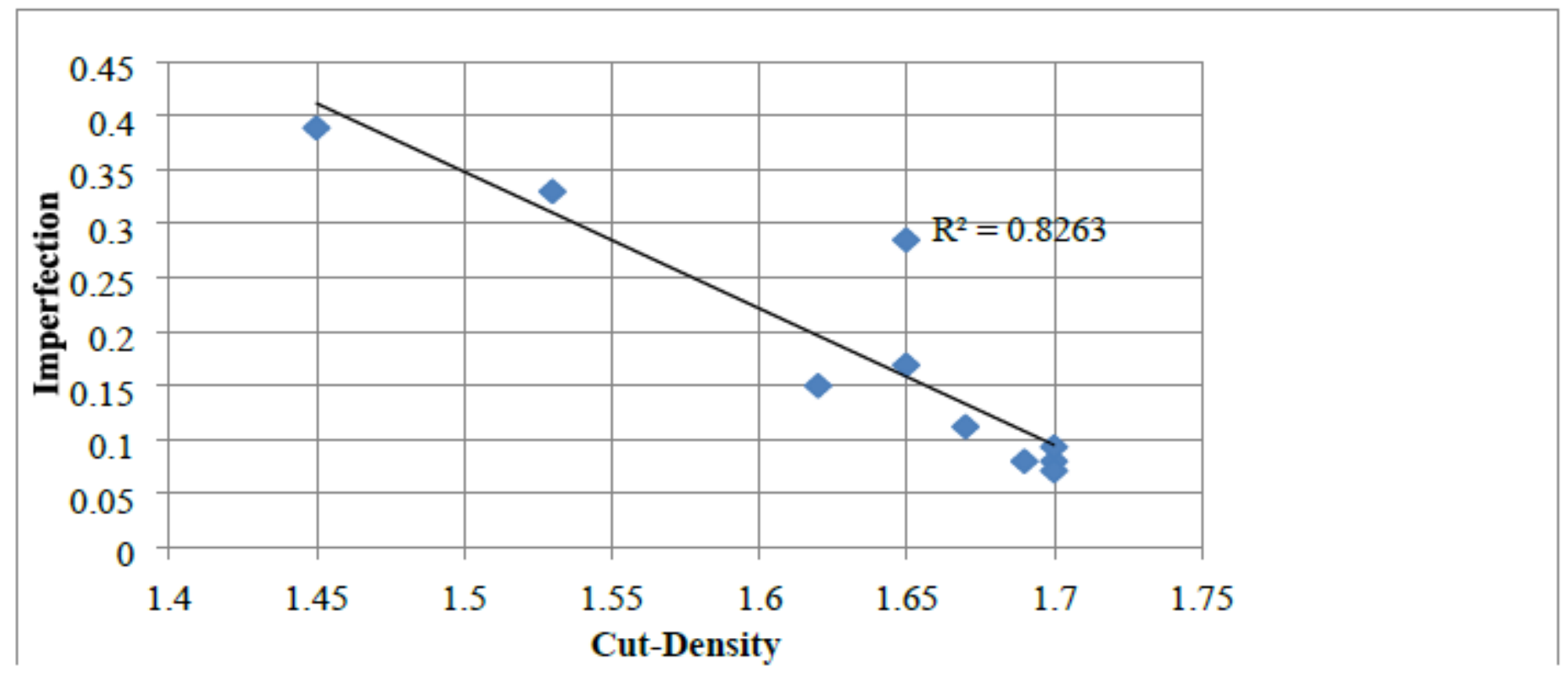

Figure 5

Dependency of imperfection on cut-density for Chasnala Batac Jig 


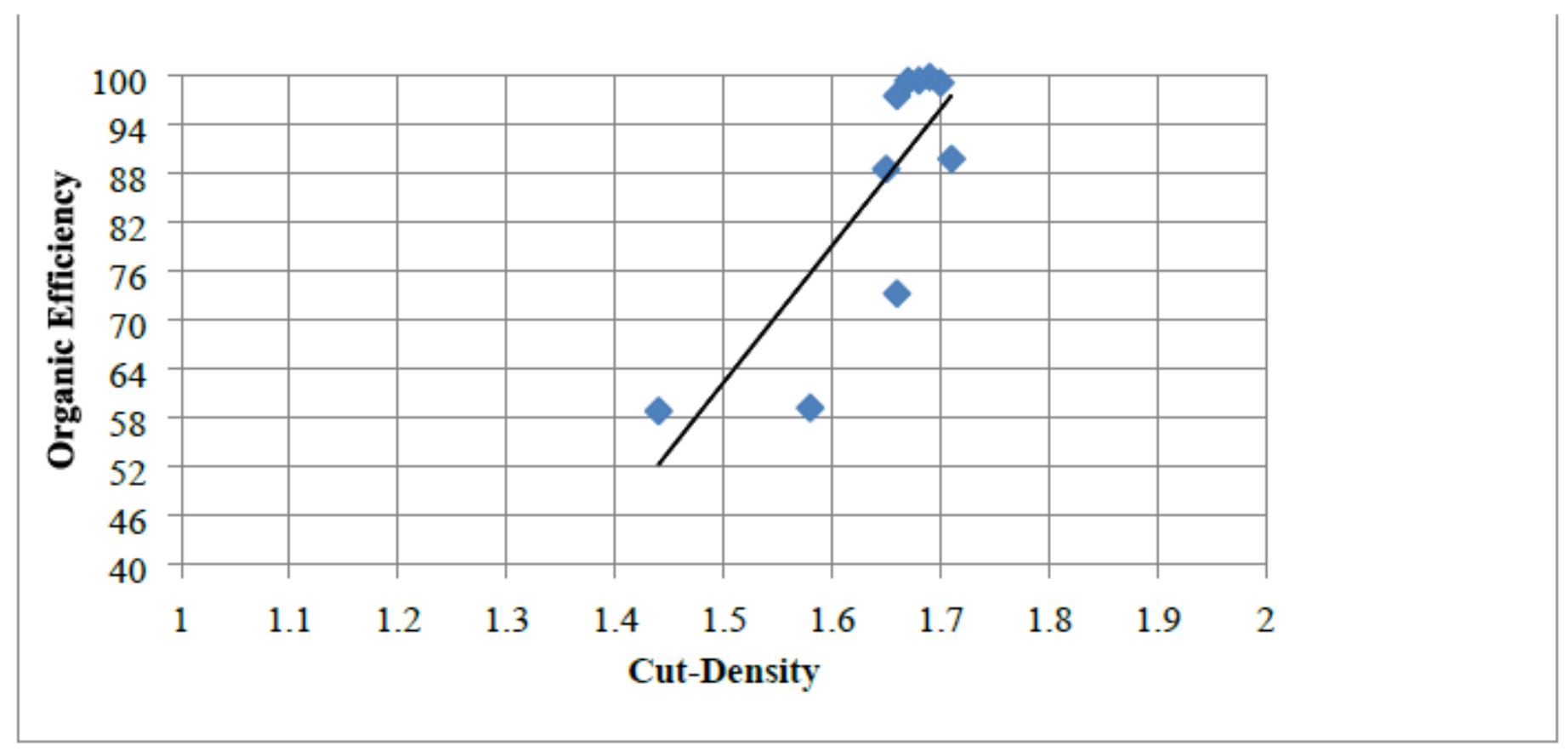

Figure 6

Dependency of organic efficiency on cut-density for Chasnala Batac Jig

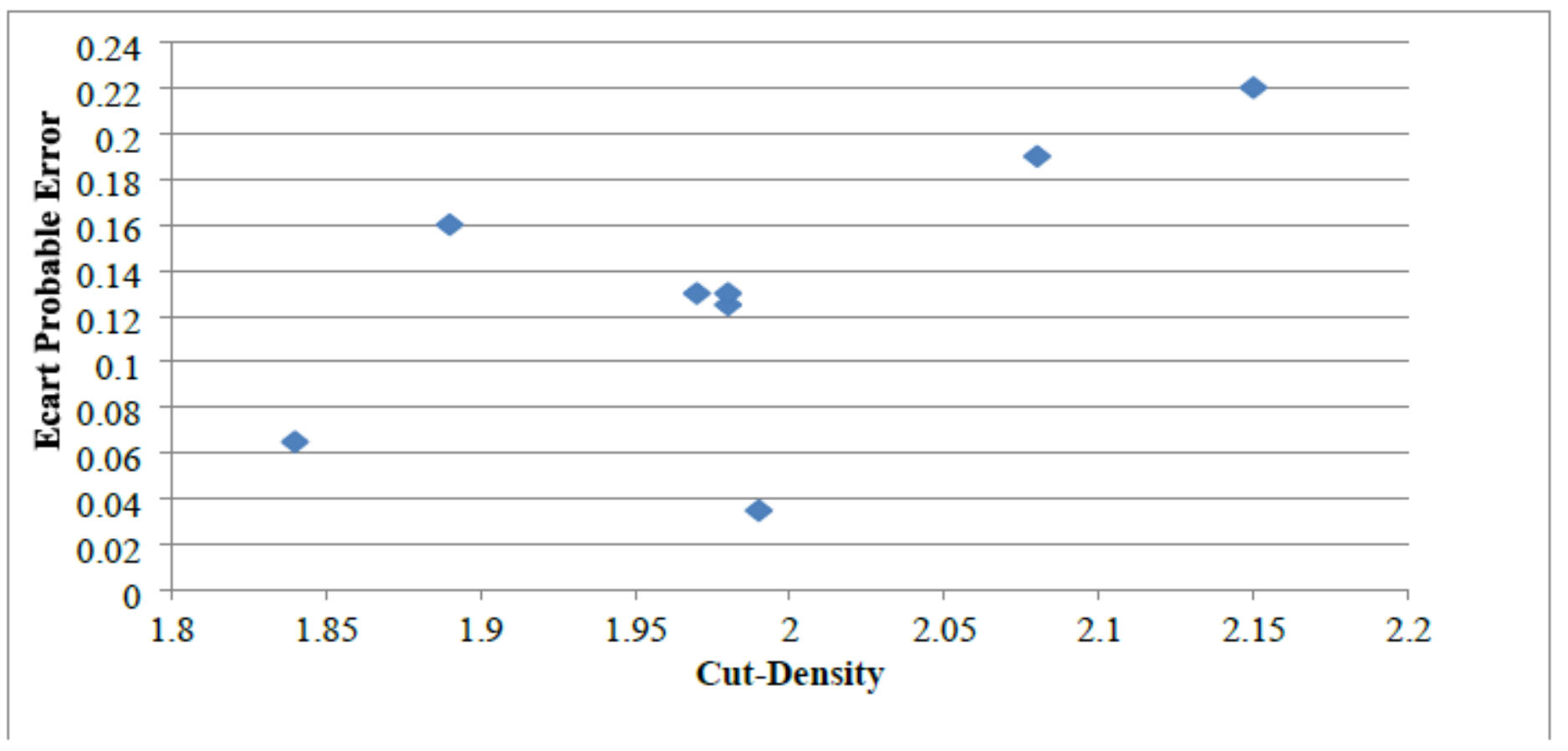

Figure 7

Plot between probable error in separation and cut-density for Sharshetali Batac Jig: Coal 1 


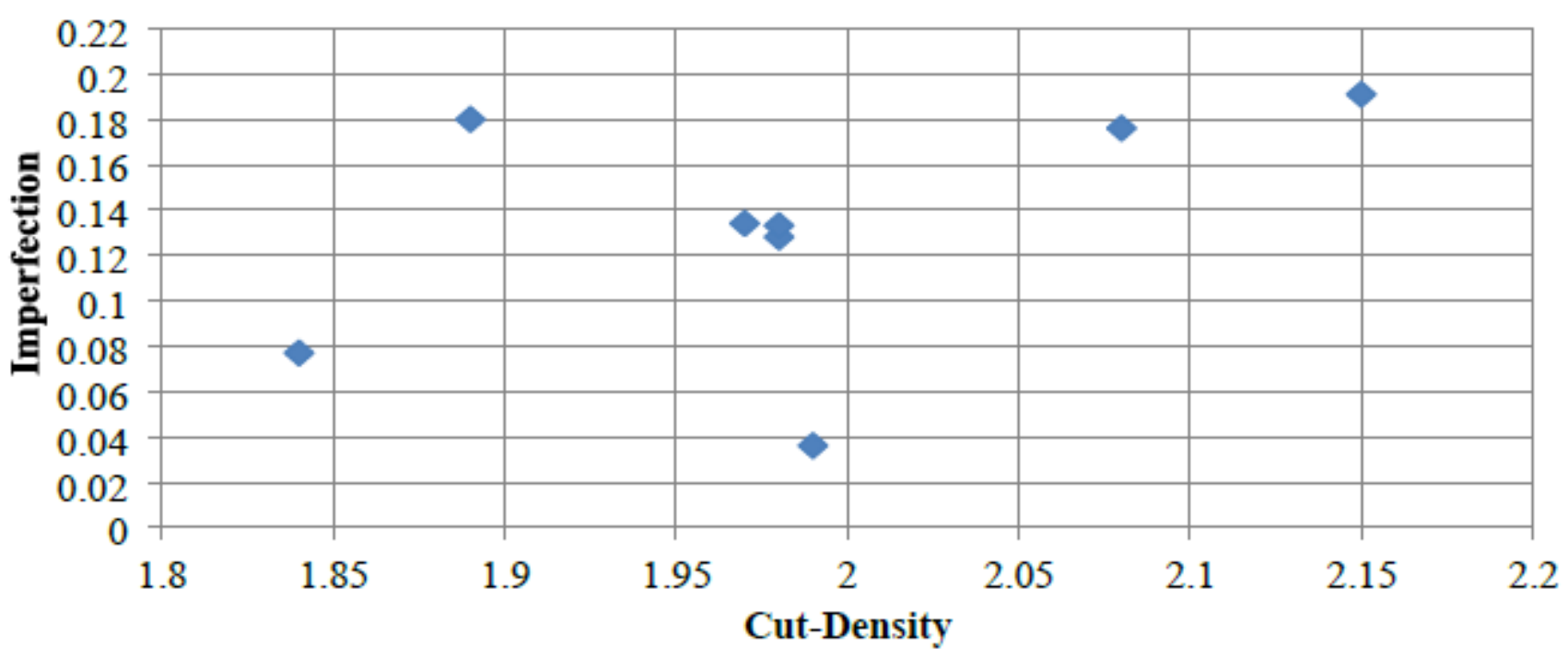

\section{Figure 8}

Plot between imperfection and cut-density for Sharshetali Batac Jig: Coal 1

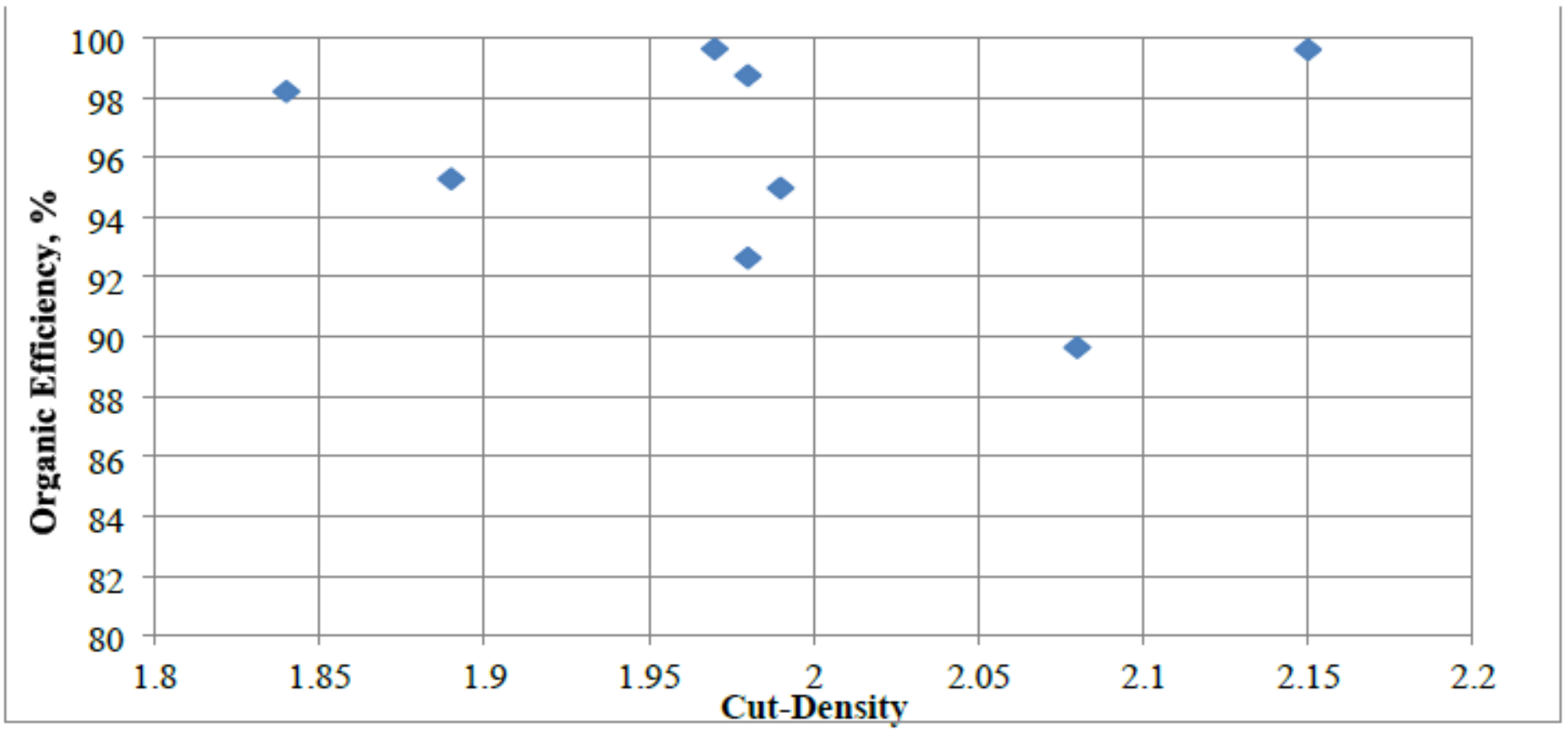

Figure 9

Plot between organic efficiency and cut-density for Sharshetali Batac Jig: Coal 1 


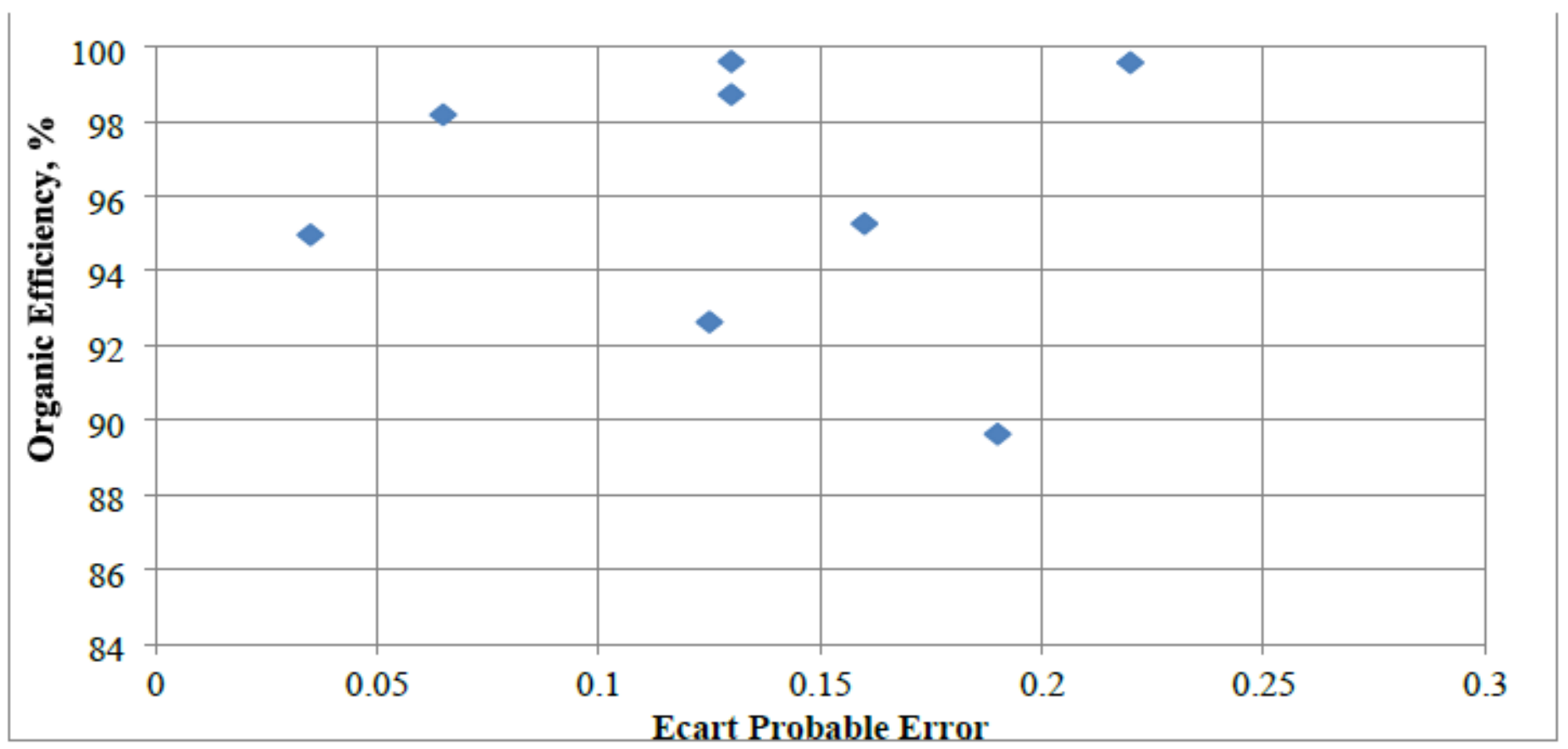

Figure 10

Plot between organic efficiency and Ep for Sharshetali Batac Jig: Coal 1

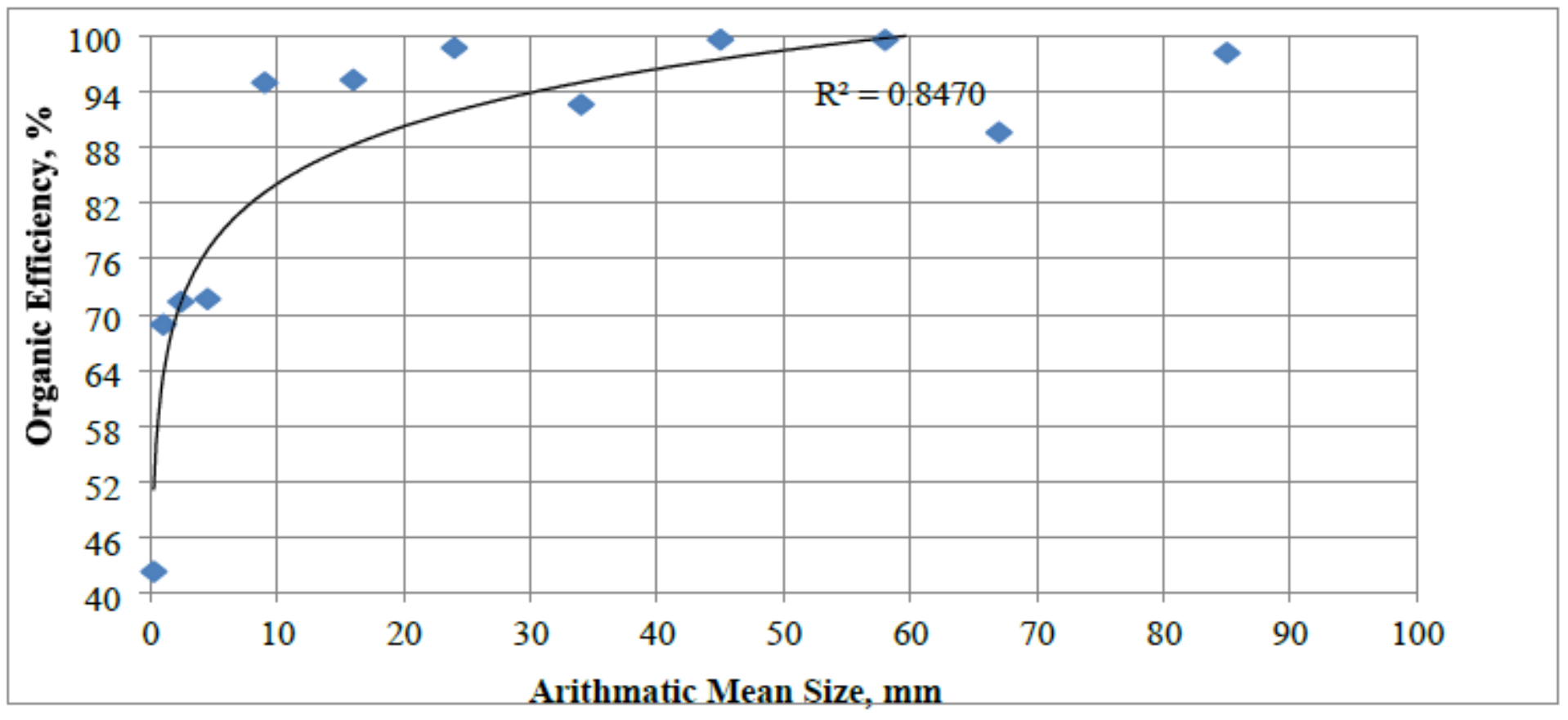

\section{Figure 11}

Size by size organic efficiencies for Sharshetali Batac Jig: Coal 2 


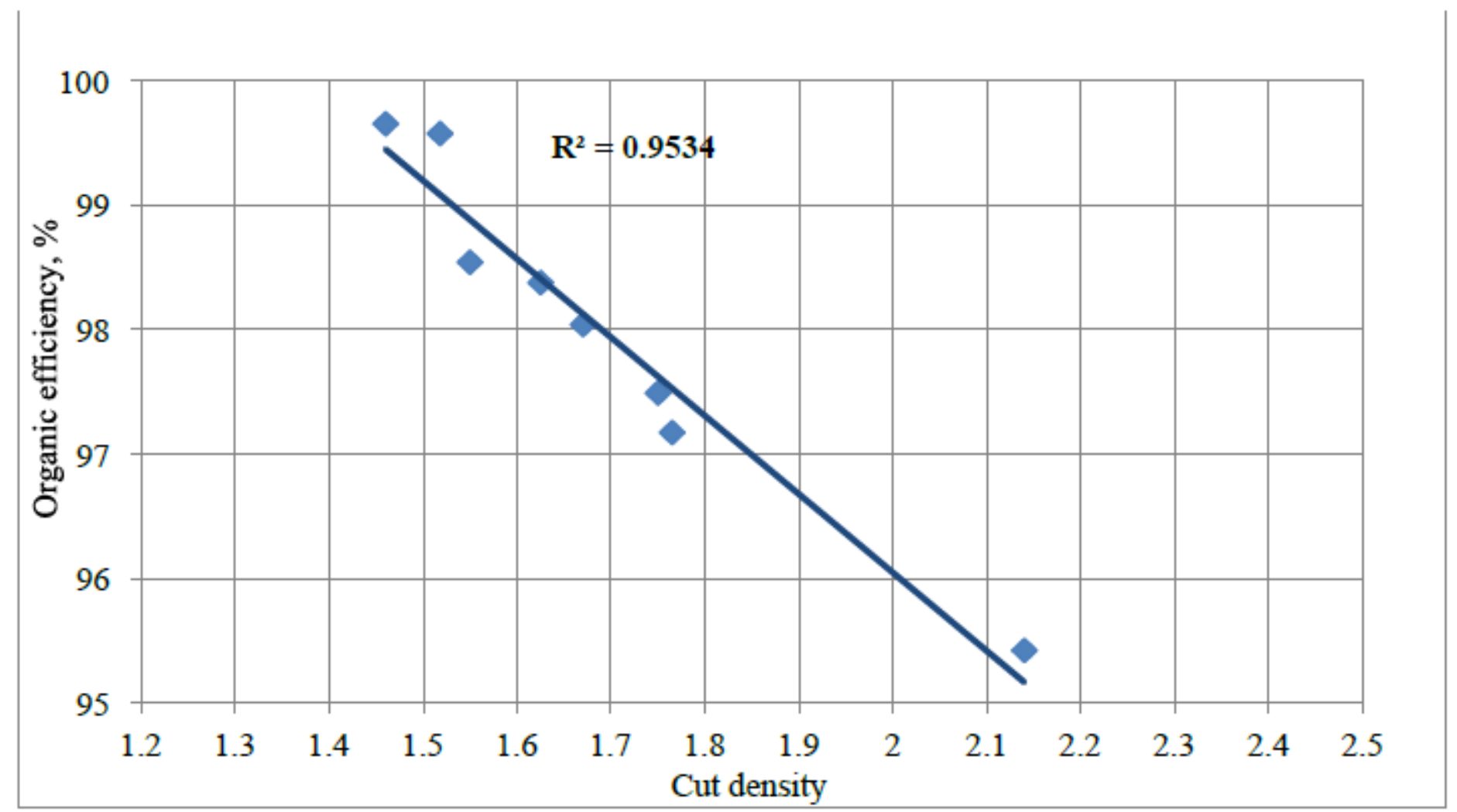

Figure 12

Dependency of organic efficiency on cut-density for the Bhojudih Leebar bath [Behera, Bhattacharya, 2018]

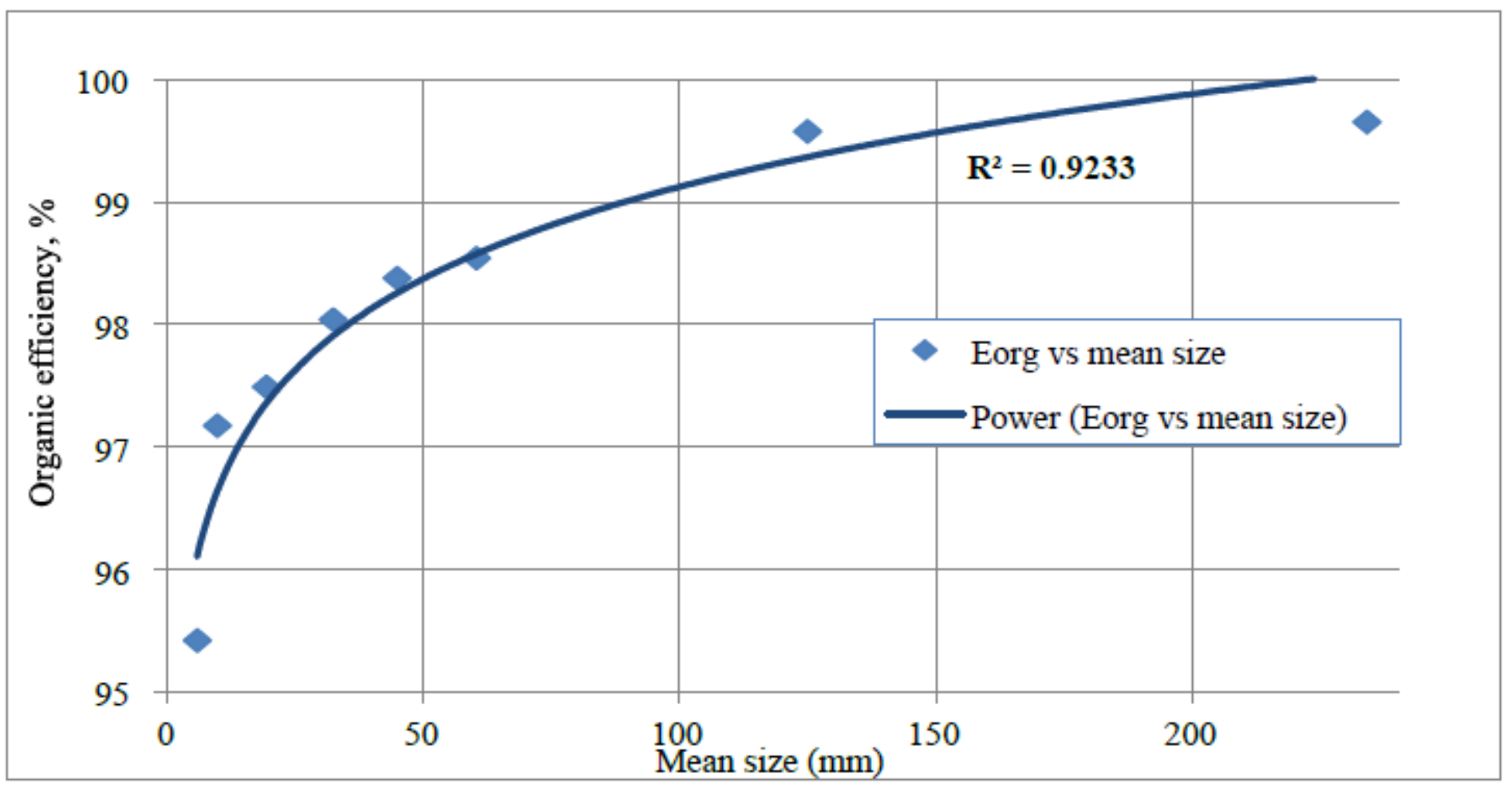

Figure 13 
Dependency of organic efficiency on particle size for the Bhojudih Leebar bath [Behera, Bhattacharya, 2018]

\section{Supplementary Files}

This is a list of supplementary files associated with this preprint. Click to download.

- Tables.pdf 